\title{
On Destructive Superposition of Shaping Pulses in Band-Limited Linear Modulation Systems
}

\author{
Makoto Tanahashi and Hideki Ochiai \\ Department of Electrical and Computer Engineering, Yokohama National University \\ Yokohama, Kanagawa 240-8501, Japan \\ Email: makoto@ochiailab.dnj.ynu.ac.jp; hideki@ynu.ac.jp
}

\begin{abstract}
We analyze pulse-shaped transmit signals with correlated input sequences in the framework of band-limited liner modulations. In general, these signals are formed by superposition (convolution) of the shaping pulses and may have different amplitude statistics depending on the correlation properties of the input sequences. Hence, even if the sequences have a unit average power in discrete-time domain, the resulting transmit signals exhibit smaller (or larger) average power if there are many destructive (or constructive) superpositions produced by the correlated source. Since such a reduction of average transmit power does not change the amplitude of the underlying discretetime sequence, one may expect the so-called shaping gain. We comprehensively analyze the shaping gain achieved by the correlation of the input sequences, and show that the use of a pulse shape, which convolves the sequence destructively, is capable of increasing the achievable information rate of the system.
\end{abstract}

\section{INTRODUCTION}

We consider a linear modulation system where an input symbol sequence is correlated due to a certain reason such as residual redundancy of incomplete source coding. In such a case, depending on the correlation property and the impulse response of the pulse-shaping filter (transmit filter), the transmitted signal contains unequal numbers of destructive (i.e., out-of-phase) and constructive (i.e., in-phase) superpositions of the shaping pulses. Hence, even if the baseline signal constellation is a phase shift keying (PSK) which always has a unit energy, the transmit signal exhibits a smaller (or larger) average power if there are many destructive (or constructive) superpositions introduced by the correlated source. This observation is illustrated in Fig. 1 where pulse-shaping is applied to three BPSK sequences having an identical amplitude but different auto-correlation properties. (The parameters used for these examples will be explained in later sections).

Since the average power of the pulse-shaped signals is decreased (or increased) for a fixed constellation energy, this in turn boosts (or decreases) the utilizable average energy of a discrete symbol for a given average power of pulse-shaped signals. Hence, reduction (or increase) of average transmit power, introduced by the correlation of the input, can be treated as a gain (or loss). We refer to this gain as shaping gain $\eta_{s}$ and it is related with the average power of the pulse-shaped signal $P_{a v}$ as

$$
\eta_{s}=\frac{P_{a v}^{\mathrm{uncor}}}{P_{a v}}
$$

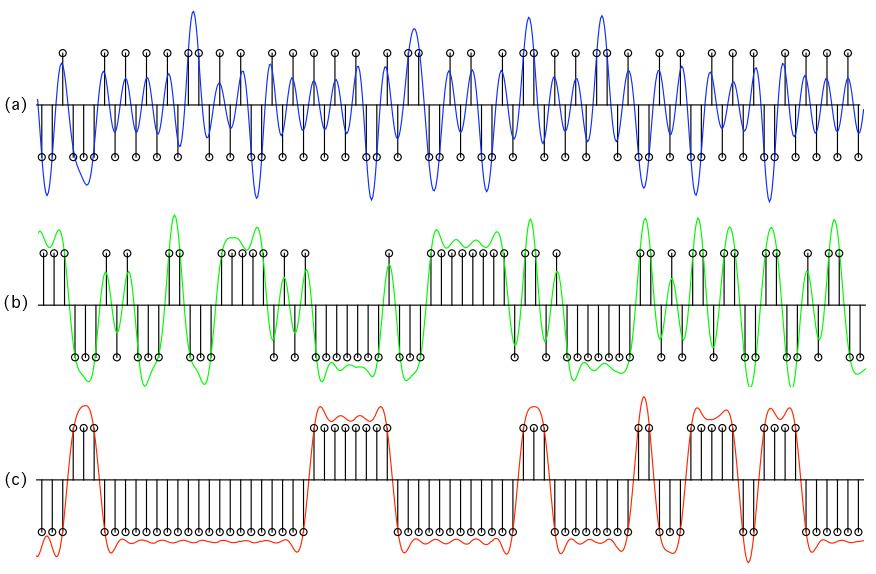

Fig. 1. Three examples of transmit signals pulse-shaped by a certain transmit filter. They have different average powers due to different correlation properties of input sequences. (a) $q=0.8$ and $\eta_{s}=2.44 \mathrm{~dB}$ (D-shaping). (b) $q=0.5$ (uncorrelated) and $\eta_{s}= \pm 0 \mathrm{~dB}$. (c) $q=0.2$ and $\eta_{s}=-1.10 \mathrm{~dB}$ (C-shaping).

where $P_{a v}^{\text {uncor }}$ denotes the average power with respect to uncorrelated input sequences. Note that the conventional definition of the shaping gain refers to constellation shaping [1], whereas the shaping gain in our definition is based on the signals after pulse shaping, i.e., the final form of the transmitted signal. One of the contributions of this paper is to mathematically analyze the average power and derive a necessary condition on the pulse-shaping filter to achieve the shaping gain.

Our analysis reveals that the shaping gain is achievable only when the filter does not satisfy the root Nyquist condition; in other words, commonly used root raised-cosine (RRC) filters cannot achieve the shaping gain regardless of the correlation property of the source. We refer to a pulse shaping as destructive pulse shaping (D-shaping) when the resulting filter matched to a given source correlation introduces many destructive superpositions (and thus yielding shaping gain). The opposite case, where the pulses are convolved constructively, is called constructive pulse shaping (C-shaping).

Since these filters do not satisfy the root Nyquist condition, the channel inevitably presents inter-symbol interference (ISI) if we presume matched filter reception. Moreover, since this ISI occurs destructively (or constructively) for the D (or C)shaping cases, the noise margin between sequences is decreased (or increased). Therefore, the achievable information 


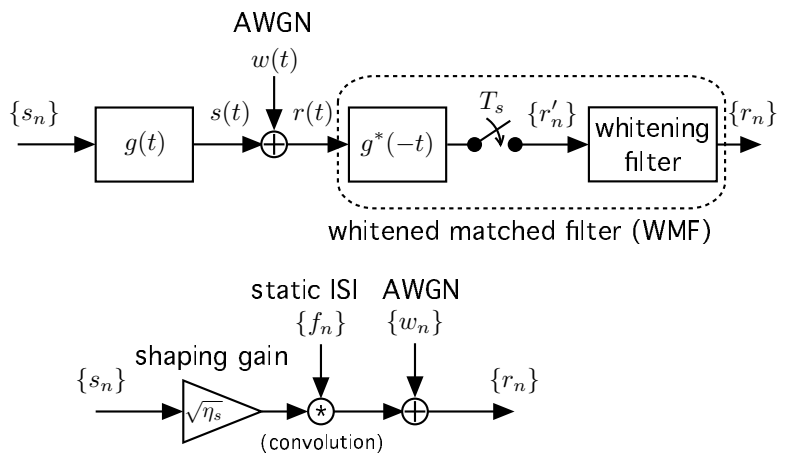

Fig. 2. Channel model and the equivalent discrete-time representation with the shaping gain $\eta_{s}$ incorporated.

rate of this ISI channel with the presence of AWGN and the shaping gain taken into account is of primary concern. To calculate this quantitatively, we follow a novel numerical technique developed independently by Arnold et al. [2] and several other researchers $[3,4]$. Interestingly, the results show that the achievable information rate of D-shaping lies above that of the conventional Nyquist filtering system, whereas Cshaping cannot surpass the Nyquist system.

The rest of this paper is organized as follows: we describe our system model in the next section. Section III shows several theorems with respect to the average power of pulse-shaped signals. Section IV presents a simple way to design filters for D- and C-shapings. An analysis on the achievable information rate is given in Section $\mathrm{V}$ and numerical results are presented in Section VI. Finally, Section VII summarizes the findings of this paper.

\section{SySTEM MOdEL}

\section{A. Continuous-Time Model}

Figure 2 shows the system model considered in this paper. In this figure, $\left\{s_{n}\right\} \triangleq S$ denotes a discrete-time sequence with the average energy (per symbol) of $E_{s}$. We also use a notation $S_{m}^{n}=\left\{s_{m}, \ldots, s_{n}\right\}$ to specify the start and end indices. (The same rule applies to other sequences). For band-limitation, $\left\{s_{n}\right\}$ is fed into a pulse-shaping filter $g(t)$ with Nyquist rate $T_{s}$ to form the transmit signal:

$$
s(t)=\sum_{n=-\infty}^{\infty} s_{n} g\left(t-n T_{s}\right) .
$$

We assume that the energy of the impulse response $g(t)$ is normalized such that $\int_{-\infty}^{\infty}|g(t)|^{2} d t=1$, and it has finite length, i.e., $\lim _{t \rightarrow \infty} g(t)=\lim _{t \rightarrow-\infty} g(t)=0$.

The considered channel-impairment between the transmitter and the receiver is only AWGN $w(t)$ with the two-sided noise power density of $N_{0} / 2$. The receiver first performs matched filtering on the received signal $r(t)=s(t)+w(t)$, and its output (after sampled with the interval $T_{s}$ ) is fed into a noisewhitening filter [5,6]. Thus, the final output $\left\{r_{n}\right\} \triangleq R$ has maximized signal-to-noise ratio as well as whitened noise which is analytically tractable.

\section{B. Equivalent Discrete-Time Model}

It is convenient for the subsequent analyses to develop an equivalent discrete-time channel model and incorporate the shaping gain $\eta_{s}$ (to be produced by $\mathrm{D}$ - or C-shapings) as a channel gain. This discrete-time channel model is shown in the lower half of Fig. 2. In this channel, the input $\left\{s_{n}\right\}$ is first amplified by $\sqrt{\eta_{s}}$ and then convolved with a static ISI $\left\{f_{n}\right\}$ which is the combined impulse response of the matched filter and the whitening filter, i.e., whitened matched filter (WMF) $[5,6]$. Adding discrete-time AWGN samples $\left\{w_{n}\right\} \triangleq W$ to this yields the channel output $\left\{r_{n}\right\}$.

The ISI coefficients $\left\{f_{n}\right\}$ can be calculated from a given $g(t)$ as follows [6]. Let $\phi_{g}[l]$ be the compound impulse response of the matched filters and the subsequent sampler, which is identical to the auto-correlation function (ACF) of $g(t)$ sampled at $l T_{s}$ and thus expressed as

$$
\phi_{g}[l]=\int_{-\infty}^{\infty} g^{*}(t) g\left(t+l T_{s}\right) d t .
$$

We suppose that the effective duration of $\phi_{g}[l]$ spans only over $2 L+1$, i.e.,

$$
\phi_{g}[l]=0, \quad \text { for } \quad|l|>L .
$$

The Z-transform of $\phi_{g}[l]$, which is given by

$$
\Phi_{g}(z)=\sum_{l=-L}^{L} \phi_{g}[l] z^{-l},
$$

has $L$ roots inside the unit circle, namely $\rho_{1}, \ldots, \rho_{L}$. Let $F(z)$ be the polynomial of degree $L$ having these roots:

$$
F(z)=\left(z-\rho_{1}\right) \cdots\left(z-\rho_{L}\right) .
$$

Then the inverse Z-transform of $F(z)$ gives the desired coefficients $\left\{f_{n}\right\}$ of the length $L+1$.

With the obtained $\left\{f_{n}\right\}$, the input-output relationship can be formulated as

$$
r_{n}=\sqrt{\eta_{s}} \sum_{l=0}^{L} s_{n-l} f_{l}+w_{n} .
$$

Our goal in this paper is to find the average mutual information (AMI) of the channel (7) for given settings of stochastic model of $\left\{s_{n}\right\}$ and pulse-shaping filter $g(t)$. This is addressed in Section V. In the next section, we start with mathematical derivation of the average transmit power.

\section{Average Transmit Power}

\section{A. Theorems for Average Transmit Power}

Theorem 1: The average power $P_{a v}$ of the signal (2) is given by

$$
P_{a v}=\frac{E_{s}}{T_{s}}\left(1+2 \sum_{l=1}^{L} \operatorname{Re}\left\{\phi_{s}[l] \phi_{g}[l]\right\}\right),
$$

where $\phi_{s}[l]=\mathrm{E}\left[s_{n+l}^{*} s_{n}\right] / E_{s}$ denotes the normalized ACF of $\left\{s_{n}\right\}$ and $\phi_{g}[l]$ is the ACF of $g(t)$ defined in (3). 

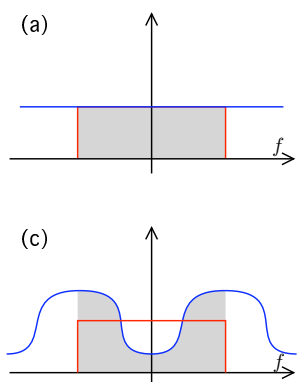
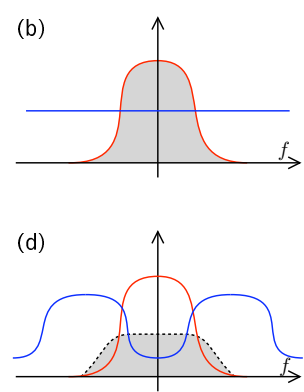

Fig. 3. Frequency domain explanation of Theorem 1 and Corollary 2. The red lines denote the PSD of the transmit filter and the blues denote that of the input sequence. The shaded areas are the product of these two PSDs. (a) uncorrelated source and root-Nyquist filter. (b) uncorrelated source and nonroot-Nyquist filter. (c) correlated source and root-Nyquist filter. (d) correlated source and non-root-Nyquist filter.

Proof: We omit the proof due to space limitation, but the readers are referred to [7]: generalization of the theorem given in Appendix of [7] easily leads to the above theorem.

The above theorem suggests that the average power depends on the correlation properties of the input sequence $\left\{s_{n}\right\}$ and the shaping pulse $g(t)$, which corroborates intuitive observation. For some particular cases, the following corollary can be derived from Theorem 1 .

Corollary 2: If either or both the following conditions is satisfied, then the average power is always given by

$$
P_{a v}=\frac{E_{s}}{T_{s}} .
$$

- The employed transmit filter $g(t)$ is a root-Nyquist filter.

- The input sequence $\left\{s_{n}\right\}$ is uncorrelated.

Proof: The first condition suggests that $\phi_{g}[n]=\delta[n]$, i.e., the Kronecker delta function, and thus the summation in (8) goes to zero. The second condition is equivalent to $\phi_{s}[n]=\delta[n]$, which leads to the same result.

The propositions above can be more intuitively explained in the frequency domain (see Fig. 3): it is known that the power spectrum density (PSD) of $s(t)$ is given by the product of the individual PSDs of $g(t)$ and $\left\{s_{n}\right\}$ [6]. The shape of each PSD fluctuates depending on parameters such as the source correlation and the filter's shape, but the areas under the PSD curves always remain constant. As a result, the PSD of $s(t)$ has a constant area if at least either $g(t)$ or $\left\{s_{n}\right\}$ has flat PSD, as illustrated in (a), (b), and (c) of the figure. On the contrary, the area and hence the average power varies if the both are not flat as shown in (d). The example of (d) corresponds to Dshaping where $\left\{s_{n}\right\}$ and $g(t)$ convolve destructively to make the summation in (8) negative.

\section{B. Realizable $\phi_{g}[l]$}

Let us suppose that the input sequence $\left\{s_{n}\right\}$ is binary and has a highest correlation such as

$$
\phi_{s}[l]= \begin{cases}+1, & l \text { is even } \\ -1, & l \text { is odd }\end{cases}
$$

In this case, if the pulse-shaping filter had the ACF of, e.g.,

$$
\left\{\phi_{g}[1], \phi_{g}[2], \phi_{g}[3]\right\}=\{0.3,-0.2,0.05\},
$$

then the average power $P_{a v}$ given by Theorem 1 would be a negative value. To guarantee such a negative average power never to arise, we have the following theorem as to realizable $\phi_{g}[l]$.

Theorem 3: There is no filter $g(t)$ that satisfies either

$$
\begin{array}{r}
-\sum_{l=1,3,5, \ldots} \phi_{g}[l]+\sum_{l=2,4,6, \ldots} \phi_{g}[l]<-\frac{1}{2}, \\
\sum_{l=1,2,3,4,5,6, \ldots} \phi_{g}[l]<-\frac{1}{2} .
\end{array}
$$

Note that the second inequality in the above theorem is to deny another highest correlation case of

$$
\phi_{s}[l]=+1, \quad \text { for all } l .
$$

\section{Design of Pulse-Shaping Filter}

In the previous section, we have shown that, in order to control the average transmission power $P_{a v}$, a pulse-shaping filter with the ACF $\phi_{g}[l]$ matched to a given correlation property $\phi_{s}[l]$ of the input sequence $\left\{s_{n}\right\}$ is required. In this section, we present how to design a pulse-shaping filter that has an arbitrary shape (within Theorem 3) of ACF. Note that, for simplicity, the impulse response $g(t)$ is restricted to be a real function. Furthermore, we deal with the sampled version $g[n] \triangleq g(n \Delta t)$ instead of $g(t)$ itself, where $\Delta t=T_{s} / N_{s}$ for an over-sampling factor $N_{s}$.

Fig. 4 illustrates the design process by using an example ACF $\phi_{g}[l]$ of $L=2$ :

$$
\left\{\phi_{g}[1], \phi_{g}[2]\right\}=\{0.3,-0.1\} \text {. }
$$

Firstly, these entries are set every $N_{s}$ as shown in Fig. 4 (a). Note that, by definition, $\phi_{g}[0]=1$. Since the length of this array determines the length of $g[n]$, the last entry of the $\mathrm{ACF}$, which is $\phi_{g}[2]$ in this example, can be followed by an arbitrary number of zeros. Next, applying FFT to this time series, we transform it into the frequency domain and then multiply an ideal low-pass filter (ILPF) to obtain the PSD of the desired filter of Fig. 4 (b). This is further transformed into an amplitude-spectrum by taking the square root as in Fig. 4 (c). Finally, the application of IFFT and an appropriate cyclic shift gives the desired impulse response $g[n]$ as shown in Fig. 4 (d). Note that a full raised-cosine filter can substitute for the ILPF so that the resulting impulse response has a shorter sustain time.

\section{D-SHAPING AND C-SHAPING}

\section{A. Stochastic Model of $\left\{s_{n}\right\}$}

For the subsequent analyses, we consider a stationary binary first-order Markov model for $\left\{s_{n}\right\}$. This Markov process moves from one to another state with the transition probability $q$. The state transition matrix is thus defined as

$$
Q=\left[\begin{array}{cc}
1-q & q \\
q & 1-q
\end{array}\right] .
$$



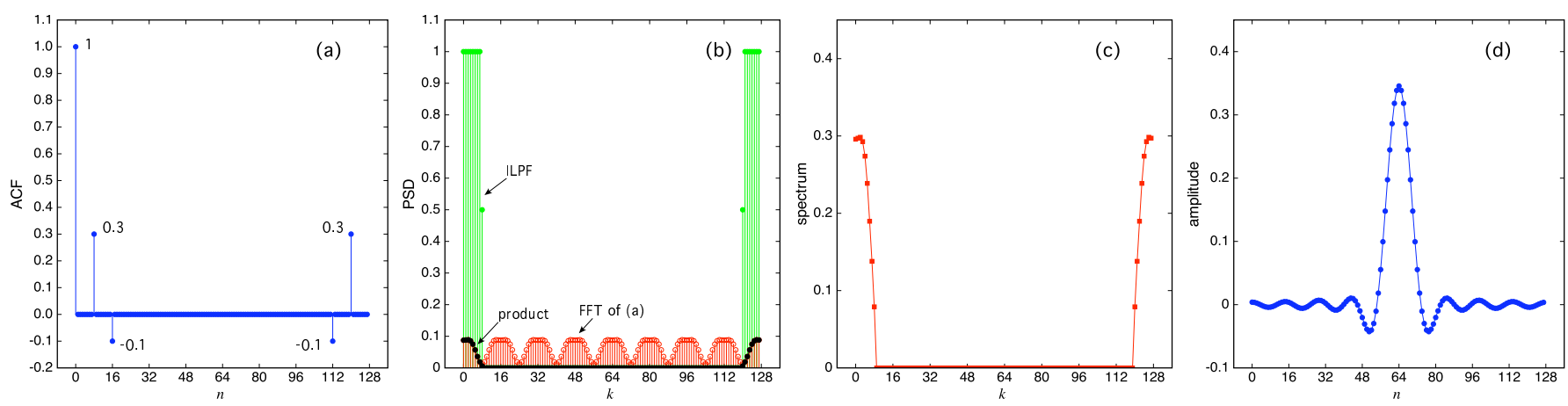

Fig. 4. Design of pulse-shaping filter from a given shape of the ACF. $N_{s}=8$.

We further define that $s_{n}=+\sqrt{E_{s}}$ if the $n$th state is 0 and $s_{n}=-\sqrt{E_{s}}$ for the $n$th state of 1 . With some algebra, one can find that the ACF $\phi_{s}[l]$ for the above settings is given by

$$
\phi_{s}[l]=\operatorname{det}\left(Q^{l}\right), \quad l=1,2,3, \ldots
$$

We observe that the possible polarities of this ACF, important to design the filter's ACF $\phi_{g}[l]$, are classified as

$$
\operatorname{sign}\left(\phi_{s}[1], \phi_{s}[2], \ldots\right)= \begin{cases}-,+,-,+, \ldots, & q>0.5 \\ 0,0,0,0, \ldots, & q=0.5 \\ +,+,+,+, \ldots, & q<0.5\end{cases}
$$

\section{B. Pulse-Shaping Filter}

The following three pulse-shaping filters, referred to as Filter-I $(L=1)$, Filter-II $(L=1)$, and Filter-III $(L=2)$, are considered:

Filter-I: $\quad\left\{\phi_{g}[1]\right\}=\{0.2\}$,

Filter-II: $\quad\left\{\phi_{g}[1]\right\}=\{-0.2\}$,

Filter-III: $\left\{\phi_{g}[1], \phi_{g}[2]\right\}=\{0.3,-0.1\}$ (same as (15)).

From Theorem 1 and (18), it is clear that the combination of $\left\{s_{n}\right\}$ with $q>0.5$ and Filter-I or Filter-III results in Dshaping, whereas the combination with Filter-II behaves as Cshaping. The destructivity and constructivity change when $q<$ 0.5 and a pulse shaping is neutral when $q=0.5$. Moreover, we can now analytically calculate the shaping gain (1) as

$$
\eta_{s}=\left(1+2 \sum_{l=1}^{L} \operatorname{det}\left(Q^{l}\right) \phi_{g}[l]\right)^{-1} .
$$

Note that, since both $\phi_{s}[l]$ and $\phi_{g}[l]$ are real-valued, the operator Re has been omitted.

The examples that we showed in Fig. 1 (at the first page) are the signals shaped by Filter-III, with three different Markov transition probabilities $q=0.8,0.5$, and 0.2 . We have confirmed that the shaping gain calculated with (22) and that actually computed via a simulation match exactly.
The discrete-time channel taps calculated with the procedure in Section II-B are, respectively,

$$
\text { Filter-I: } \quad\left\{f_{0}, f_{1}\right\}=\{0.98,0.20\}
$$

Filter-II: $\quad\left\{f_{0}, f_{1}\right\}=\{-0.98,0.20\}$,

Filter-III: $\quad\left\{f_{0}, f_{1}, f_{2}\right\}=\{-0.92,-0.37,0.11\}$.

A commonly used ISI-free matched filter system, operating with a pair of root raised-cosine (RRC) filters, corresponds to $f_{0}=1(L=0)$ and always $\eta_{s}=1$. We also consider this system for comparison.

\section{Average Mutual Information}

Having specified the stochastic model of $\left\{s_{n}\right\}$ and the channel taps $\left\{f_{n}\right\}$, we now proceed to evaluate the AMI of the channel (7).

First, let us express the AMI $I(S ; R)$ in terms of (differential) entropies as

$$
I(S ; R)=h(R)-h(R \mid S)=h(R)-h(W) .
$$

The second term, denoting the entropy of a white Gaussian noise with variance $N_{0} / 2$, is given by the closed form of $h(W)=\frac{1}{2} \log _{2}\left(\pi e N_{0}\right)$. On the other hand, the first term can be numerically calculated by means of the forward-recursion procedure similar to the BCJR algorithm [2]. Specifically,

$$
h(R) \rightarrow \frac{1}{n} \log _{2} p\left(R_{1}^{n}\right)
$$

holds as $n \rightarrow \infty$. In [2], it is reported that $n=10^{6}$ is sufficient for the convergence. To compute the $n$-dimensional pdf $p\left(R_{1}^{n}\right)$ with practical effort, we rewrite it as

$$
p\left(R_{1}^{n}\right)=\sum_{\text {all possible } \Gamma_{n}} p\left(R_{1}^{n}, \Gamma_{n}\right),
$$

where the state $\Gamma_{n}$ is defined as

$$
\Gamma_{n} \triangleq\left[s_{n}, s_{n-1}, \ldots, s_{n-L^{\prime}}\right],
$$

for $L^{\prime}=\max (L, 1)$. In (28), if we define $\alpha_{n} \triangleq p\left(R_{1}^{n}\right)$, it then 


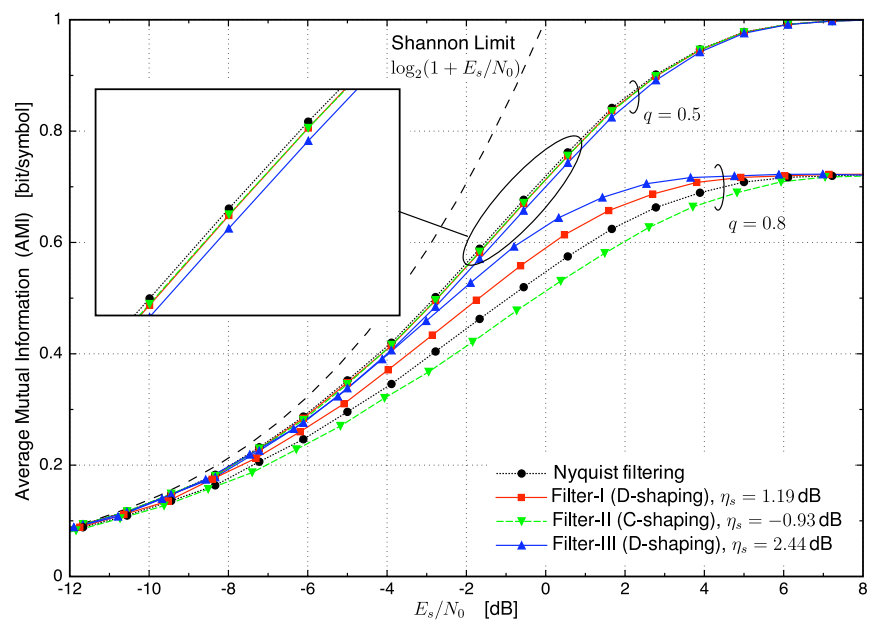

Fig. 5. AMI vs. $E_{s} / N_{0}$ for the considered filters and the transition probabilities $q=0.8$

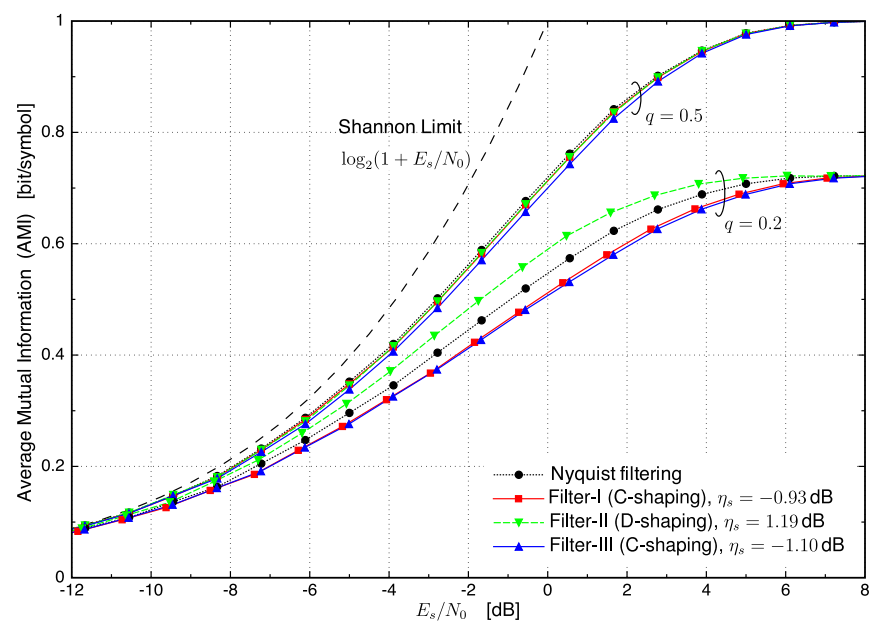

Fig. 6. AMI vs. $E_{s} / N_{0}$ in the case of $q=0.2$.

satisfies the following recursive equation:

$$
\begin{gathered}
\alpha_{n}=\sum_{\text {all possible } \Gamma_{n-1}} p\left(r_{n} \mid \Gamma_{n}, \Gamma_{n-1}\right) \operatorname{Pr}\left(\Gamma_{n} \mid \Gamma_{n-1}\right) \alpha_{n-1} \\
=\sum_{\text {all possible } \Gamma_{n-1}} p_{\mathrm{G}}\left(\sqrt{\eta_{s}} \sum_{l=0}^{L} s_{n-l} f_{l}-r_{n}\right) \\
\cdot P_{t}\left(s_{n-1} \rightarrow s_{n}\right) \alpha_{n-1},
\end{gathered}
$$

where $p_{\mathrm{G}}(\cdot)$ denotes the Gaussian pdf with the variance $N_{0} / 2$. The Markov transition probability $P_{t}\left(s_{n-1} \rightarrow s_{n}\right)$ equals to $q$ if $s_{n} \neq s_{n-1}$ and is $1-q$ otherwise. By evaluating (30) for $\left\{s_{n}\right\}$ and $\left\{r_{n}\right\}$ generated with a simulation, we can obtain the desired AMI.

\section{RESUlTS AND Discussions}

Figures 5 and 6 show the AMI as a function of the SNR, $E_{s} / N_{0}$, for two Markov transition probability settings $q=0.8$ and 0.2. Thus, as mentioned in Section V-B, Filters-I and III behave as D-shaping for $q=0.8$ (or, C-shaping for 0.2 ) and the opposite result applies to Filter-II. The associated shaping gains (or, losses) are also displayed in the figures. From these results, the following remarks can be made.

- For the uncorrelated case (i.e., $q=0.5$ ), the conventional Nyquist filtering maximizes the AMI. However, interestingly, D-shaping outperforms the Nyquist filtering when the input $\left\{s_{n}\right\}$ is correlated. C-shaping, on the other hand, performs worse.

- Examining the gap between the AMI curves, we find that, if the shaping gain were neglected by shifting the curves right by $\eta_{s}$, C-shaping in turn surpassed the Nyquist filtering. This is because the sequences generated with C-shaping are amplified by the channel ISI. However, the penalty in terms of the average power overuse (i.e., shaping loss) is more significant than the improvement achieved with the constructive ISI. As a result, the actual AMI of C-shaping lies below that of the Nyquist system.

- Filter-III achieves a larger shaping gain than that of Filter-I in the D-shaping scenario, and Filter-III also has a greater AMI even though it suffers from a severer destructive ISI in the channel. From this result, we conjecture that the maximization of the AMI can be achieved by designing a transmit filter so as to maximize the achievable shaping gain based on (22).

\section{CONCLUSION}

We have shown that if the source is correlated due to a certain reason such as residual redundancy of incomplete source coding, one can improve the achievable information rate (i.e., AMI) by intentionally introducing D-shaping at the transmitter. In this sense, D-shaping can be seen as a precoding. The distinguishing feature of this precoding lies in that it compensates for source correlation as opposed to channel memory (i.e., ISI). Designing a channel code that achieves the derived AMI appears to be challenging: the insertion of a channel code before D-shaping affects the correlation property of the source. In this regard, the existing capacity-approaching codes such as turbo and LDPC codes do not appear to be preferable, as the random-interleaving nature of these codes collapses the correlation, meaning that D-shaping has no effect.

\section{REFERENCES}

[1] R. F. H. Fischer, Precoding and Signal Shaping for Digital Transmission. John Wiley \& Sons Inc, 2002.

[2] D. M. Arnold, H. A. Loeliger, P. O. Vontobel, A. Kavčić, and W. Zeng, "Simulation-based computation of information rates for channels with memory," IEEE Trans. Inform. Theory, vol. 52, pp. 3498-3508, Aug. 2006.

[3] H. D. Pfister, J. B. Soriaga, and P. H. Siegel, "On the achievable information rates of finite state ISI channels," in Proc. IEEE Globecom'01, pp. 2992-2996, Nov. 2001.

[4] V. Sharma and S. K. Singh, "Entropy and channel capacity in the regenerative setup with applications to Markov channels," in Proc. IEEE ISIT'01, p. 283, June 2001.

[5] G. D. Forney, Jr., "Maximum-likelihood sequence estimation of digital sequences in the presence of intersymbol interference," IEEE Trans. Inform. Theory, vol. 18, pp. 363-378, May 1972.

[6] J. G. Proakis, Digital Communications. McGrawHill, 4th ed., 2001.

[7] M. Tanahashi and H. Ochiai, "Near constant envelope trellis shaping for PSK signaling," IEEE Trans. Commun., vol. 57, pp. 450-458, Feb. 2009. 